\title{
Quantum states of electromagnetic field interacting with a classical current and their applications to radiation problems.
}

\author{
V. G. Bagrov ${ }^{1,2 *}$, D. M. Gitman ${ }^{3,4 \dagger}$, A. A. Shishmarev ${ }^{2 \ddagger}$ and A. J. D. Farias Jr ${ }^{4 \S}$ \\ ${ }^{1}$ Department of Physics, Tomsk State University, Lenin Prospekt 36, 634050, Tomsk, Russia; \\ 2 Institute of High Current Electronics, SB RAS, Akademichesky Ave. 4, 634055, Tomsk, Russia; \\ ${ }^{3}$ P.N. Lebedev Physical Institute, 53 Leninskiy prospect, 119991 Moscow, Russia; \\ ${ }^{4}$ Institute of Physics, University of São Paulo, \\ Rua do Matão, 1371, CEP 05508-090, São Paulo, SP, Brazil
}

March 25, 2020

\begin{abstract}
In the beginning, the synchrotron radiation (SR) was studied by classical methods using the LiénardWiechert potentials of electric currents. Subsequently, quantum corrections to the obtained classical formulas were studied, considering the emission of photons arising from electronic transitions between spectral levels, described in terms of the Dirac equation. In this paper, we consider an intermediate approach, in which electric currents generating the radiation are considered classically, whereas the quantum nature of the radiation is taken into account exactly. Such an approximate approach may be helpful in some cases, it allows one to study the one-photon and multi-photon radiation without complicating calculations using corresponding solutions of the Dirac equation. We construct exact quantum states of the electromagnetic field interacting with classical currents and study their properties. By their help, we calculate a probability of photon emission by classical currents and obtain relatively simple formulas for the one-photon and multiphoton radiation. Using the specific circular electric current, we calculate the corresponding SR. We discuss a relation of obtained results with known before, for example, with the Schott formula, with the Schwinger calculations, with one-photon radiation of scalar particles due to transitions between Landau levels, and with some previous results of calculating the two-photon SR.

Keywords: Synchrotron radiation, multiphoton radiation
\end{abstract}

\section{Introduction}

As a rule, a motion of charged particles in external electromagnetic fields is accompanied by electromagnetic radiation. The most important examples, at the same time related to the present work, are synchrotron (SR) and cyclotron $(\mathrm{CR})$ radiations of charged particles in a magnetic field. The phenomenon of SR was discovered approximately 70 years ago [1. A large number of works have been devoted to their theoretical description, both within the framework of classical and quantum theory. In both cases, various approximate methods and limiting cases were considered. In classical electrodynamics the electromagnetic field created by an arbitrary electric four-current is described by potentials of Liénard-Wiechert (LW) [2, 3]. It turns out that SR can be described sufficiently precise in the framework of the classical theory (using LW potentials). Schott was the first to obtain a successful formula for the angular distribution of power emitted in SR by a particle moving in a circular orbit [4. An alternative derivation of classical formulas describing the properties of SR and their deep analysis, especially for high-energy relativistic electrons, was given by Schwinger [5]. Nevertheless, quantum effects may play an important role in the SR and CR. In particular, effects of a backreaction related to a photon radiation, aspects of discrete structure of energy levels of electrons in the magnetic field, and spin properties of charged particles are ignored by the classical theory. The essence of quantum corrections to classical results was first pointed out in Ref. [6]. In quantum theory, radiation rate of energy of a charge particle in course of quantum transitions was calculated using exact solutions of the Schrödinger (nonrelativistic case), Klein-Gordon (spinless case) or Dirac (relativistic case) equations with a magnetic field [7]. Using his source theory [8] Schwinger had presented an original derivation of similar results [9]. Besides, the quantum treatment revealed a completely new effect of self-polarization of electrons and positrons moving in uniform and constant magnetic field [10]. We note that in the latter works only a one-photon radiation in course of quantum transitions was taken into account. However, there are evidences that a multi-photon emission can contribute significantly to the SR, see for example [11, 12. For electromagnetic fields exceeding the critical Schwinger field $H_{0}=m^{2} c^{3} / e \hbar$, nonlinear

\footnotetext{
*bagrov@phys.tsu.ru

$\dagger$ gitman@if.usp.br

†a.a.shishmarev@mail.ru

$\S$ a.jorgedantas@gmail.com
} 
phenomena of quantum electrodynamics begin to play a prominent role. Moreover, at fields comparable with the critical field one can observe nonlinear quantum effects caused by ultrarelativistic particles with high enough momenta. Some examples of such effects (of the orders of $\alpha, \alpha^{2}$ ( $\alpha$ is a fine-structure constant) in the interaction with the radiation field) are the one-photon emission by electrons $(e \rightarrow e \gamma, \alpha)$, the pair production by photons $\left(\gamma \rightarrow e^{+} e^{-}, \alpha\right)$, electron scattering accompanied by the pair production $\left(e \rightarrow e e^{+} e^{-}, \alpha^{2}\right)$, two photon emission process $\left(e \rightarrow e 2 \gamma, \alpha^{2}\right)$ etc. If an incident particle has a momentum $p \sim\left(H_{0} / H\right) m$, then the probability of the processes is notable.

It should be noted a significant complexity of calculating even the one-photon radiation using solutions of the above mentioned quantum equations. There is an opportunity to simplify these calculations considering in the same relatively simple manner the multi-photon radiation taking the quantum nature of the irradiated field into account exactly, but considering the particle current classically. This means that we neglect the back reaction of the radiation to the current that generates this radiation. Such an approximation may be justified in some cases, for example, for high density electron beams. From the technical point of view, this means that calculating electromagnetic radiation induced by classical electric currents, we have to work with exact quantum states of the electromagnetic field interacting with classical currents. Such an approach is considered in the present work. For these purposes, we first construct exact quantum states of the electromagnetic field interacting with classical currents and study their properties. Then by their help, we calculate a probability of photons emission by a classical current from the vacuum initial state (i.e., from the state without initial photons). Then we obtain relatively simple formulas for one-photon and multi-photon radiation. Using the specific circular electric current, we calculate the corresponding SR. We discuss a relation of obtained results with known before, for example, with the Schott formula, with the Schwinger calculations, with one-photon radiation of scalar particles due to transitions between Landau levels, and with some known results of calculating the two-photon SR. Less important technical details are placed in the Appendix.

\section{Quantum states of the radiation field interacting with a classical current}

Here we consider the quantized electromagnetic field interacting with a classical current $J_{\mu}(x)$, see Refs. [13. 14, 15, 16, 17. In the Coulomb gauge this system is described by a Hamiltonian $\hat{H}$ which consists of two terms, a Hamiltonian of free transversal photons $\hat{H}_{\gamma}$ and an interaction Hamiltonian $\hat{H}_{\text {int }}$ :

$$
\begin{aligned}
& \hat{H}=\hat{H}_{\gamma}+\hat{H}_{\mathrm{int}}, \hat{H}_{\gamma}=c \hbar \sum_{\lambda=1,2} \int d \mathbf{k} k_{0} \hat{c}_{\mathbf{k} \lambda}^{\dagger} \hat{c}_{\mathbf{k} \lambda}, \\
& \hat{H}_{\mathrm{int}}=\frac{1}{c} \int\left[J_{i}(x) \hat{A}^{i}(\mathbf{r})+\frac{1}{2} J_{0}(x) A^{0}(x)\right] d \mathbf{r} .
\end{aligned}
$$

Here $\hat{A}^{i}(\mathbf{r})$ are operators (in the Schrödinger representation) of vector potentials of the transversal electromagnetic field,

$$
\begin{aligned}
& \hat{A}^{i}(\mathbf{r})=\sqrt{4 \pi c \hbar} \sum_{\lambda=1}^{2} \int d \mathbf{k}\left[\hat{c}_{\mathbf{k} \lambda} f_{\mathbf{k} \lambda}^{i}(\mathbf{r})+\hat{c}_{\mathbf{k} \lambda}^{\dagger} f_{\mathbf{k} \lambda}^{i *}(\mathbf{r})\right], i=1,2,3, \\
& f_{\mathbf{k} \lambda}^{i}(\mathbf{r})=\frac{\exp (i \mathbf{k r})}{\sqrt{2 k_{0}(2 \pi)^{3}}} \epsilon_{\mathbf{k} \lambda}^{i}, \quad k_{0}=|\mathbf{k}|,
\end{aligned}
$$

where $\epsilon_{\mathbf{k} \lambda}^{i}$ are the polarization vectors of the photon with wave vector $\mathbf{k}$ and polarization $\lambda=1,2$. These vectors possess the properties

$$
\epsilon_{\mathbf{k} \lambda} \epsilon_{\mathbf{k} \sigma}^{*}=\delta_{\lambda \sigma}, \epsilon_{\mathbf{k} \lambda} \mathbf{k}=0, \sum_{\lambda=1}^{2} \epsilon_{\mathbf{k} \lambda}^{i} \epsilon_{\mathbf{k} \lambda}^{j *}=\delta^{i j}-\frac{k^{i} k^{j}}{|\mathbf{k}|^{2}} .
$$

Operators $\hat{c}_{\mathbf{k} \lambda}$ and $\hat{c}_{\mathbf{k} \lambda}^{\dagger}$ are the annihilation and creation operators of photons with a wave vector $\mathbf{k}$ and polarizations $\lambda$. These operators satisfy the commutation relations:

$$
\left[\hat{c}_{\mathbf{k} \lambda}, \hat{c}_{\mathbf{k}^{\prime} \lambda^{\prime}}^{\dagger}\right]=\delta_{\lambda \lambda^{\prime}} \delta\left(\mathbf{k}-\mathbf{k}^{\prime}\right),\left[\hat{c}_{\mathbf{k} \lambda}, \hat{c}_{\mathbf{k}^{\prime} \lambda^{\prime}}\right]=\left[\hat{c}_{\mathbf{k} \lambda}^{\dagger}, \hat{c}_{\mathbf{k}^{\prime} \lambda^{\prime}}^{\dagger}\right]=0
$$

Using Eqs. (2)-(4) one can verify that the operator $\hat{A}^{i}(\mathbf{r})$ satisfy the condition $\operatorname{div} \hat{\mathbf{A}}(\mathbf{r})=0$. We note that in the Coulomb gauge $A^{0}(x)$ is a $c$-valued scalar function which satisfies the following equations:

$$
A^{0}(x)=\int d \mathbf{r}^{\prime} \frac{J_{0}\left(\mathbf{r}^{\prime}, t\right)}{\left|\mathbf{r}-\mathbf{r}^{\prime}\right|}, \Delta A^{0}(x)=-4 \pi J_{0}(x) .
$$

Then the term $J_{0}(x) A^{0}(x) / 2$ can be represented as $-2 \pi J_{0}(x) \Delta^{-1} J_{0}(x)$, and, in general case, is time dependent. 
The evolution of state vectors $|\Psi(t)\rangle$ of the quantized electromagnetic field is governed by the Schrödinger equation

$$
i \hbar \partial_{t}|\Psi(t)\rangle=\hat{H}|\Psi(t)\rangle .
$$

The general solution of Eq. (7) can be written in the following form, 1 ,

$$
\begin{aligned}
& |\Psi(t)\rangle=U(t)|\Psi(0)\rangle \\
& U(t)=\exp \left[-i \hbar^{-1} \hat{H}_{\gamma} t\right] \exp \left[-i \hbar^{-1} \hat{B}(t)\right] \\
& \hat{B}(t)=\frac{1}{c} \int_{0}^{t} d t^{\prime} \int\left\{J_{i}\left(x^{\prime}\right)\left[\hat{A}^{i}\left(x^{\prime}\right)+\frac{1}{2} \tilde{A}^{i}\left(x^{\prime}\right)\right]+\frac{1}{2} J_{0}\left(x^{\prime}\right) A^{0}\left(x^{\prime}\right)\right\} d \mathbf{r}^{\prime}, \\
& \tilde{A}^{i}(x)=\frac{1}{\hbar c} \int_{0}^{t} d t^{\prime} \int D_{0}\left(x-x^{\prime}\right) \delta_{\perp}^{i k} J^{k}\left(x^{\prime}\right) d \mathbf{r}^{\prime}, \\
& \hat{A}^{i}(x)=\sqrt{4 \pi c \hbar} \sum_{\lambda=1}^{2} \int d \mathbf{k}\left[\hat{c}_{\mathbf{k} \lambda} f_{\mathbf{k} \lambda}^{i}(x)+\hat{c}_{\mathbf{k} \lambda}^{\dagger} f_{\mathbf{k} \lambda}^{i *}(x)\right], f_{\mathbf{k} \lambda}^{i}(x)=f_{\mathbf{k} \lambda}^{i}(\mathbf{r}) e^{-i k_{0} c t},
\end{aligned}
$$

where $U(t)$ is an evolution operator, and $|\Psi(0)\rangle$ is an initial state of the quantized electromagnetic field at the time instant $t=0$.

The singular function $D_{0}\left(x-x^{\prime}\right)$ can be obtained from the Pauli-Jordan permutation function at $m=0$, see, for example, [20],

$$
\square D_{0}\left(x-x^{\prime}\right)=0, D_{0}\left(x-x^{\prime}\right)=4 \pi c \hbar \frac{i}{(2 \pi)^{3}} \int \frac{d \mathbf{k}}{2 k_{0}}\left[e^{-i k\left(x-x^{\prime}\right)}-e^{i k\left(x-x^{\prime}\right)}\right] .
$$

It defines nonequal-time commutation relations for the operators $\hat{A}^{i}(x)$,

$$
\left[\hat{A}^{i}(x), \hat{A}^{j}\left(x^{\prime}\right)\right]=-i \delta_{\perp}^{i j} D_{0}\left(x-x^{\prime}\right), \delta_{\perp}^{i j}=\delta^{i j}-\Delta^{-1} \partial^{i} \partial^{j}
$$

and is related to the retarded $D^{\text {ret }}\left(x-x^{\prime}\right)$ and advanced $D^{\text {adv }}\left(x-x^{\prime}\right)$ Green's functions of the D'Alembert equations,

$$
\begin{aligned}
& \int_{0}^{t} d t^{\prime} D_{0}\left(x-x^{\prime}\right)=\int_{0}^{\infty} d t^{\prime} D^{\mathrm{ret}}\left(x-x^{\prime}\right), D_{0}\left(x-x^{\prime}\right)=D^{\mathrm{ret}}\left(x-x^{\prime}\right)-D^{\mathrm{adv}}\left(x-x^{\prime}\right), \\
& D^{\mathrm{ret}}\left(x-x^{\prime}\right)=\theta\left(t-t^{\prime}\right) D_{0}\left(x-x^{\prime}\right), D^{\mathrm{adv}}\left(x-x^{\prime}\right)=\theta\left(t^{\prime}-t\right) D_{0}\left(x-x^{\prime}\right), \\
& \square D^{\mathrm{ret}}\left(x-x^{\prime}\right)=\square D^{\mathrm{adv}}\left(x-x^{\prime}\right)=\delta\left(x-x^{\prime}\right) .
\end{aligned}
$$

Taking into account Eqs. (12), one can see that the functions $\tilde{A}^{i}(x)$ represent retarded potentials created by a classical current (see, e.g., [2, 21]).

Let us verify directly that state vector (8) satisfies equation (77). Foremost, as the operator $\hat{H}_{\gamma}$ is timeindependent, we have

$$
i \hbar \partial_{t}\left[\exp \left(-i \hbar^{-1} \hat{H}_{\gamma} t\right)\right]=\hat{H}_{\gamma} \exp \left(-i \hbar^{-1} \hat{H}_{\gamma} t\right)
$$

However, the derivative $\partial_{t} \hat{A}^{i}(x)$ does not commute with the operators $\hat{A}^{i}\left(x^{\prime}\right)$, so when calculating the derivative $i \hbar \partial_{t}$ of the second exponent in the RHS of Eq. (9), one has to use the Feynman's method of disentangling of operators 22]. Calculating the derivative $i \hbar \partial_{t}$ in such a way, we find

$$
\begin{aligned}
& i \hbar \partial_{t} \exp \left[-i \hbar^{-1} \hat{B}(t)\right]=\hat{K}(t) \exp [-i \hat{B}(t)], \hat{K}(t)=\int_{0}^{1} d s e^{-i s \hbar^{-1} \hat{B}(t)}\left[\partial_{t} \hat{B}(t)\right] e^{i s \hbar^{-1} \hat{B}(t)}, \\
& \partial_{t} \hat{B}(t)=\frac{1}{c} \int\left\{J_{i}(x)\left[\hat{A}^{i}(x)+\frac{1}{2} \tilde{A}^{i}(x)\right]+\frac{1}{2} J_{0}(x) A^{0}(x)\right\} d \mathbf{r} .
\end{aligned}
$$

Using the operator relation

$$
e^{\hat{A}} \hat{M} e^{-\hat{A}}=\hat{M}+[\hat{A}, \hat{M}]+\frac{1}{2 !}[\hat{A},[\hat{A}, \hat{M}]]+\ldots,
$$

we represent the integrand in the RHS of $\hat{K}(t)$ as follows:

$$
\begin{aligned}
& e^{-i s \hbar^{-1} \hat{B}(t)}\left[\partial_{t} \hat{B}(t)\right] e^{i s \hbar^{-1} \hat{B}(t)}=\partial_{t} \hat{B}(t)+\left[-i s \hbar^{-1} \hat{B}(t), \partial_{t} \hat{B}(t)\right] \\
& +\frac{1}{2 !}\left[-i s \hbar^{-1} \hat{B}(t),\left[-i s \hbar^{-1} \hat{B}(t), \partial_{t} \hat{B}(t)\right]\right]+\ldots
\end{aligned}
$$

\footnotetext{
${ }^{1}$ See Refs. [18, 19].
} 
Calculating the first commutator in this series, we obtain:

$$
\left[\hat{B}(t), \partial_{t} \hat{B}(t)\right]=\frac{1}{c^{2}} \int_{0}^{t} d t^{\prime} \iint\left\{J_{i}\left(x^{\prime}\right)\left[\hat{A}^{i}\left(x^{\prime}\right), \hat{A}^{j}(x)\right] J_{j}(x)\right\} d \mathbf{r} d \mathbf{r}^{\prime} .
$$

The nonequal-time commutation relations for the operators $\hat{A}^{i}(x)$ are given by Eq. (11). Then (16) takes the form

$$
\left[\hat{B}(t), \partial_{t} \hat{B}(t)\right]=-\frac{i}{c^{2}} \int_{0}^{t} d t^{\prime} \int d \mathbf{r} J_{j}(x) \int d \mathbf{r}^{\prime} J_{i}\left(x^{\prime}\right) \delta_{\perp}^{i j} D_{0}\left(x-x^{\prime}\right) .
$$

We suppose, as usual, that currents under consideration vanish at spatial infinities. In this case,

$$
\int d \mathbf{r}^{\prime} J_{i}\left(x^{\prime}\right) \delta_{\perp}^{i j} D_{0}\left(x-x^{\prime}\right)=\int d \mathbf{r}^{\prime} D_{0}\left(x-x^{\prime}\right) \delta_{\perp}^{i j} J_{i}\left(x^{\prime}\right) .
$$

Then, recalling the definition of $\tilde{A}^{i}(x)$ from the evolution operator (9), we obtain

$$
\left[\hat{B}(t), \partial_{t} \hat{B}(t)\right]=-\frac{i}{c} \int J_{i}(x) \tilde{A}^{i}(x) d \mathbf{r} .
$$

Since the right-hand side of Eq. (19) is not an operator, the only first commutator in the RHS of Eq. (15) survives. Substituting Eqs. (14), (15) and (19) into Eq. (14) and then integrating over $s$, we find:

$$
\hat{K}(t)=\frac{1}{c} \int\left[J_{i}(x) \hat{A}^{i}(x)+\frac{1}{2} J_{0}(x) A^{0}(x)\right] d \mathbf{r} .
$$

Using the fact that in the Coulomb gauge

$$
\exp \left[-i \hbar^{-1} \hat{H}_{\gamma} t\right] \hat{K}(t)=\frac{1}{c} \int\left[J_{i}(x) \hat{A}^{i}(\mathbf{r})+\frac{1}{2} J_{0}(x) A^{0}(x)\right] d \mathbf{r} \exp \left[-i \hbar^{-1} \hat{H}_{\gamma} t\right],
$$

and taking into account Eq. (13), we make sure that state vector (8) does satisfy equation (7).

It is useful to represent the evolution operator $U(t)$ as:

$$
\begin{aligned}
& U(t)=\exp [i \phi(t)] \exp \left[-i \hbar^{-1} \hat{H}_{\gamma} t\right] \mathcal{D}(y), \\
& \mathcal{D}(y)=\exp \left\{\sum_{\lambda=1}^{2} \int d \mathbf{k}\left[y_{\mathbf{k} \lambda}(t) \hat{c}_{\mathbf{k} \lambda}^{\dagger}-y_{\mathbf{k} \lambda}^{*}(t) \hat{c}_{\mathbf{k} \lambda}\right]\right\}, \\
& \phi(t)=-\frac{1}{2 c} \int_{0}^{t} d t^{\prime} \int\left[J_{i}\left(x^{\prime}\right) \tilde{A}^{i}\left(x^{\prime}\right)+J_{0}\left(x^{\prime}\right) A^{0}\left(x^{\prime}\right)\right] d \mathbf{r}^{\prime}, \\
& y_{\mathbf{k} \lambda}(t)=-i \sqrt{\frac{4 \pi}{\hbar c}} \int_{0}^{t} d t^{\prime} \int J_{i}\left(x^{\prime}\right) f_{\mathbf{k} \lambda}^{i *}\left(x^{\prime}\right) d \mathbf{r}^{\prime} .
\end{aligned}
$$

In what follows we omit the argument $(t)$ in functions $y_{\mathbf{k} \lambda}(t)$ to make formulas more compact.

We remind some basic relations for the displacement operator $\mathcal{D}(\alpha)$ in the Coulomb gauge,

$$
\begin{aligned}
& \mathcal{D}^{\dagger}(\alpha)=\mathcal{D}^{-1}(\alpha),|\alpha\rangle=\mathcal{D}(\alpha)|0\rangle, \hat{c}_{\mathbf{k} \lambda}|\alpha\rangle=\alpha_{\mathbf{k} \lambda}|\alpha\rangle, \\
& \mathcal{D}^{\dagger}(\alpha) \hat{c}_{\mathbf{k} \lambda} \mathcal{D}(\alpha)=\hat{c}_{\mathbf{k} \lambda}+\alpha_{\mathbf{k} \lambda}, \mathcal{D}^{\dagger}(\alpha) \hat{c}_{\mathbf{k} \lambda}^{\dagger} \mathcal{D}(\alpha)=\hat{c}_{\mathbf{k} \lambda}^{\dagger}+\alpha_{\mathbf{k} \lambda}^{*} .
\end{aligned}
$$

With their help, we obtain:

$$
\mathcal{D}(y)|0\rangle=\exp \left(-\frac{1}{2} \sum_{\lambda=1}^{2} \int d \mathbf{k}\left|y_{\mathbf{k} \lambda}\right|^{2}\right) \exp \left(\sum_{\lambda=1}^{2} \int d \mathbf{k} y_{\mathbf{k} \lambda} c_{\mathbf{k} \lambda}^{\dagger}\right)|0\rangle .
$$

\section{Electromagnetic radiation induced by a classical current}

One can use the constructed state vector (8) to study electromagnetic radiation induced by a classical current. For simplicity, we choose the vacuum $|0\rangle$ as the initial state $|\Psi(0)\rangle$ at the $t=0$ in Eq. (8). The time evolution of this initial state follows from the latter equation:

$$
|\Psi(t)\rangle=\exp [i \phi(t)] \exp \left[-i \hbar^{-1} \hat{H}_{\gamma} t\right] \mathcal{D}(y)|0\rangle .
$$

Using Eq. (27), we can calculate a probability of photons emission.

When operating in a continuous Fock space, see. [14, a state with $N$ photons is formed by the repeated action of the photon creation operators on the vacuum $|0\rangle$, and has the form:

$$
|\{N\}\rangle=(N !)^{-1 / 2} \prod_{i=1}^{N} \hat{c}_{\mathbf{k}_{i} \lambda_{i}}^{\dagger}|0\rangle
$$


where $\hat{c}_{\mathbf{k}_{i} \lambda_{i}}^{\dagger}$ are creation operators of photons with wave vector $\mathbf{k}_{i}$ and polarizations $\lambda_{i},\{N\}=\left(\mathbf{k}_{1} \lambda_{1}, \mathbf{k}_{2} \lambda_{2}, \ldots, \mathbf{k}_{N} \lambda_{N}\right)$.

A probability amplitude $R(\{N\}, t)$ of the transition from the vacuum state $|0\rangle$ to the state (28) for the time interval $t$ reads:

$$
R(\{N\}, t)=\exp [i \phi(t)]\left\langle 0\left|(N !)^{-1 / 2}\left(\prod_{i=1}^{N} \hat{c}_{\mathbf{k}_{i} \lambda_{i}}\right) \exp \left[-i \hat{H}_{\gamma} t\right] \mathcal{D}(y)\right| 0\right\rangle .
$$

Using properties (25) and (26) of the displacement operator $\mathcal{D}(y)$, and commutation relations (5), one can represent amplitude (29) as follows:

$$
\begin{aligned}
& R(\{N\}, t)=R(0, t)(N !)^{-1 / 2} \prod_{i=1}^{N} \exp \left[-i\left|\mathbf{k}_{i}\right| c t\right] y_{\mathbf{k}_{i} \lambda_{i}}, \\
& R(0, t)=\langle 0 \mid \Psi(t)\rangle=\exp [i \phi(t)] \exp \left(-\frac{1}{2} \sum_{\lambda=1}^{2} \int d \mathbf{k}\left|y_{\mathbf{k} \lambda}\right|^{2}\right) .
\end{aligned}
$$

Then the corresponding differential probability $P(\{N\}, t)$ of such a transition (which we interpret as differential probability of the photon emission) has the form:

$$
\begin{aligned}
& P(\{N\}, t)=|R(\{N\}, t)|^{2}=p(\{N\}, t) P(0, t), \\
& p(\{N\}, t)=(N !)^{-1} \prod_{i=1}^{N}\left|y_{\mathbf{k}_{i} \lambda_{i}}\right|^{2}, \\
& P(0, t)=|R(0, t)|^{2}=\exp \left(-\sum_{\lambda=1}^{2} \int d \mathbf{k}\left|y_{\mathbf{k} \lambda}\right|^{2}\right),
\end{aligned}
$$

where $P(0, t)$ is the vacuum-to-vacuum transition probability, or the probability of a transition without any photon emission. Thus, $p(\{N\}, t)$ is the relative probability of a process in which $N$ photons with quantum numbers $\mathbf{k}_{i} \lambda_{i}$ are emitted (the relative differential probability).

One can obtain the total probability $P(N, t)$ of transition from the vacuum state $|0\rangle$ to the state with $N$ arbitrary photons, summing the quantity $p(\{N\}, t)$ over the sets $\{N\}$. Thus, we get2:

$$
\begin{aligned}
& P(N, t)=\sum_{\{N\}} P(\{N\}, t)=P(0, t) p(N, t), \sum_{\{N\}}=\prod_{i=1}^{N}\left(\sum_{\lambda_{i}} \int d \mathbf{k}_{i}\right), \\
& p(N, t)=(N !)^{-1} \prod_{i=1}^{N}\left(\sum_{\lambda_{i}} \int d \mathbf{k}_{i}\left|y_{\mathbf{k}_{i} \lambda_{i}}\right|^{2}\right) .
\end{aligned}
$$

Introducing a total probability $P(t)$ of the photon emission for the time interval $t$ as follows

$$
P(t)=\sum_{N=1}^{\infty} P(N, t)=P(0, t) \sum_{N=1}^{\infty}(N !)^{-1} \prod_{i=1}^{N}\left(\sum_{\lambda_{i}} \int d \mathbf{k}_{i}\left|y_{\mathbf{k}_{i} \lambda_{i}}\right|^{2}\right)
$$

one can easily verify that the relation $P(0, t)+P(t)=1$ holds true.

The electromagnetic energy of $\{N\}$ photons with given quantum numbers $\{\mathbf{k} \lambda\}=\left(\mathbf{k}_{i} \lambda_{i}, i=1,2, \ldots, N\right)$ depends only on their momenta $\{\mathbf{k}\}=\left(\mathbf{k}_{i}, i=1,2, \ldots, N\right)$ and does not depend on their polarizations; it is equal to

$$
W(\{N\})=\hbar c\left[\sum_{i=1}^{N}\left|\mathbf{k}_{i}\right|\right] .
$$

Then the total electromagnetic energy $W(N, t)$ of $N$ emitted photon reads:

$$
W(N, t)=\sum_{\{N\}} W(\{N\}) p(\{N\}, t)=\hbar c(N !)^{-1} \sum_{\lambda_{1}=1}^{2} \sum_{\lambda_{2}=1}^{2} \ldots \sum_{\lambda_{N}=1}^{2} \int d \mathbf{k}_{1} d \mathbf{k}_{2} \ldots d \mathbf{k}_{N}\left[\sum_{j=1}^{N}\left|\mathbf{k}_{i}\right|\right] \prod_{i=1}^{N}\left|y_{\mathbf{k}_{i} \lambda_{i}}\right|^{2} .
$$

It is easy to demonstrate (see Appendix) that $W(N, t)$ can be represented as:

$$
\begin{aligned}
& W(N, t)=\frac{A}{(N-1) !}\left(\sum_{\lambda=1}^{2} \int d \mathbf{k}\left|y_{\mathbf{k} \lambda}\right|^{2}\right)^{N-1}, \\
& A=\hbar c \sum_{\lambda=1}^{2} \int d \mathbf{k} k_{0}\left|y_{\mathbf{k} \lambda}\right|^{2}, \quad k_{0}=|\mathbf{k}| .
\end{aligned}
$$

\footnotetext{
${ }^{2}$ It should be noted that Glauber [23] derived the total probability $P(N, t)$ by his own method, however, did not consider its application for the radiation problem.
} 
Finally, we calculate the total energy $W(t)$ of emitted photons:

$$
W(t)=\sum_{N=1}^{\infty} W(N, t)
$$

The sum (37) can be calculated exactly, taking into account Eq. (36),

$$
W(t)=A \exp \sum_{\lambda=1}^{2} \int d \mathbf{k}\left|y_{\mathbf{k} \lambda}\right|^{2}
$$

\section{One-photon radiation by a circular current}

Here we study one-photon radiation from the vacuum induced by a specific circular current. Here we are interested in calculating one-photon radiation, that is why we will discuss a probability of the appearance of one photon with given quantum numbers $\mathbf{k}$ and $\lambda=1,2$. Thus, we consider a transition amplitude from the state (27) to the final state of the form (28) with $N=1$. Using (35), we write one-photon emission as

$$
W(1, t)=\hbar c \sum_{\lambda=1}^{2} \int d \mathbf{k} k_{0}\left|y_{\mathbf{k} \lambda}\right|^{2}, k_{0}=|\mathbf{k}| .
$$

Let us consider a circular current formed by electrons moving perpendicularly to an external uniform and constant magnetic field $\mathbf{H}=(0,0, H)$ with the velocity $\mathbf{v}$ along a circular trajectory of the radius $R$. Such a current has the following form [7]:

$$
\begin{aligned}
& J_{0}(x)=q \delta^{(3)}(\mathbf{r}-\mathbf{r}(t)), \mathbf{J}(x)=q \dot{\mathbf{r}}(t) \delta^{(3)}(\mathbf{r}-\mathbf{r}(t)), \\
& \mathbf{r}(t)=(R \cos \omega t, R \sin \omega t, 0), \mathbf{v}(t)=\dot{\mathbf{r}}(t)=\omega R(-\sin \omega t, \cos \omega t, 0),
\end{aligned}
$$

where $q=-e, e>0$ is the electron charge, $\omega=e H / m c$ is the cyclotron frequency. We disregard the backreaction of the radiation, i.e., we suppose that the current is maintained in its original form during the time interval $\Delta t=t$.

Functions $y_{\mathbf{k} \lambda}(22)$ for the current (40) have the form:

$$
\begin{aligned}
& y_{\mathbf{k} \lambda}=i q \int_{0}^{t} d t^{\prime} \frac{\mathbf{v}\left(t^{\prime}\right) \epsilon_{\mathbf{k} \lambda}^{*}}{\sqrt{\hbar c k_{0}(2 \pi)^{2}}} \exp \left\{i\left[k_{0} c t^{\prime}-\mathbf{k r}\left(t^{\prime}\right)\right]\right\}, \\
& \mathbf{k}=\left(k_{\perp} \cos \varphi, k_{\perp} \sin \varphi, k_{\|}\right), k_{\perp}=k_{0} \sin \theta, k_{\|}=k_{0} \cos \theta .
\end{aligned}
$$

Here $\varphi$ is the angle between the $x$ axis and the projection of the vector $\mathbf{k}$ onto the $x y$ plane, and $\theta$ is the angle between the $z$ axis and $\mathbf{k}$. Thus,

$$
W(1, t)=\frac{\hbar c}{(2 \pi)^{2}} \int d \mathbf{k} k_{0}\left|\int d t^{\prime} \mathbf{J}\left(x^{\prime}\right) \epsilon_{\mathbf{k} \lambda}^{*} \exp \left[i k_{0} c t^{\prime}-\mathbf{k r}\left(t^{\prime}\right)\right]\right|^{2} .
$$

Then

$$
\begin{aligned}
& \exp \left[-i \mathbf{k r}\left(t^{\prime}\right)\right]=\exp \left[-i k_{\perp} R \sin \tau\right] \\
& \exp \left(i k_{0} c t^{\prime}\right)=\exp \left[i c k_{0} \omega^{-1}(\varphi-\pi / 2)\right] \exp \left(i c k_{0} \omega^{-1} \tau\right), \\
& \mathbf{v}(\tau)=\omega R[\cos (\tau+\varphi), \sin (\tau+\varphi), 0] \\
& \tau=\tau_{\mathrm{i}}+\omega t^{\prime}, \tau_{\mathrm{i}}=\pi / 2-\varphi, \int_{0}^{t} d t^{\prime} \rightarrow \int_{\tau_{\mathrm{i}}}^{\tau_{\mathrm{i}}+\omega t} \omega^{-1} d \tau
\end{aligned}
$$

In the case under consideration, we chose linear polarization vectors $\epsilon_{\mathbf{k} \lambda}$ as:

$$
\begin{aligned}
& \epsilon_{\mathbf{k} 1}=(\cos \varphi \cos \theta, \sin \varphi \cos \theta,-\sin \theta), \epsilon_{\mathbf{k} 2}=(-\sin \varphi, \cos \varphi, 0), \\
& \epsilon_{\mathbf{k} 1} \epsilon_{\mathbf{k} 1}=\epsilon_{\mathbf{k} 2} \epsilon_{\mathbf{k} 2}=1, \epsilon_{\mathbf{k} 1} \epsilon_{\mathbf{k} 2}=\epsilon_{\mathbf{k} 1} \mathbf{k}=\epsilon_{\mathbf{k} 2} \mathbf{k}=0
\end{aligned}
$$

One can easily verify that the following relations hold:

$$
\mathbf{v}\left(t^{\prime}\right) \epsilon_{\mathbf{k} 1}^{*}=\omega R \cos \theta \cos \tau, \mathbf{v}\left(t^{\prime}\right) \epsilon_{\mathbf{k} 2}^{*}=\omega R \sin \tau
$$


Now it follows from Eqs. (41) that

$$
\begin{aligned}
& y_{\mathbf{k} 1}=\frac{i q R \cos \theta}{\sqrt{k_{0}(2 \pi)^{2} \hbar c}} Y_{\mathbf{k}}(\varphi) \int_{\tau_{\mathrm{i}}}^{\tau_{\mathrm{i}}+\omega t} d \tau \exp \left(i c k_{0} \omega^{-1} \tau\right) \cos \tau \exp \left(-i k_{\perp} R \sin \tau\right), \\
& y_{\mathbf{k} 2}=\frac{i q R}{\sqrt{k_{0}(2 \pi)^{2} \hbar c}} Y_{\mathbf{k}}(\varphi) \int_{\tau_{\mathrm{i}}}^{\tau_{\mathrm{i}}+\omega t} d \tau \exp \left(i c k_{0} \omega^{-1} \tau\right) \sin \tau \exp \left(-i k_{\perp} R \sin \tau\right) \\
& Y_{\mathbf{k}}(\varphi)=\exp \left[i c k_{0} \omega^{-1}(\varphi-\pi / 2)\right] .
\end{aligned}
$$

At this stage, we utilize a well-known plane wave expansion of the Bessel functions $j_{n}(x)$ (see, e.g., [7]),

$$
\begin{aligned}
& \exp \left(-i k_{\perp} R \sin \tau\right)=\sum_{n=-\infty}^{+\infty} j_{n}\left(k_{\perp} R\right) \exp (-i n \tau), \\
& \sin \tau \exp \left(-i k_{\perp} R \sin \tau\right)=i \sum_{n=-\infty}^{+\infty} j_{n}^{\prime}\left(k_{\perp} R\right) \exp (-i n \tau), \\
& \cos \tau \exp \left(-i k_{\perp} R \sin \tau\right)=\sum_{n=-\infty}^{+\infty} \frac{n}{k_{\perp} R} j_{n}\left(k_{\perp} R\right) \exp (-i n \tau) .
\end{aligned}
$$

Using (48) in Eqs. (47), we obtain:

$$
\begin{aligned}
& y_{\mathbf{k} 1}=i \frac{q R \cos \theta}{\sqrt{k_{0}(2 \pi)^{2} \hbar c}} Y_{\mathbf{k}}(\varphi) \sum_{n=-\infty}^{+\infty} \frac{n j_{n}\left(k_{\perp} R\right)}{k_{\perp} R} F_{\mathbf{k}}^{n}(\varphi, t), \\
& y_{\mathbf{k} 2}=-\frac{q R}{\sqrt{k_{0}(2 \pi)^{2} \hbar c}} Y_{\mathbf{k}}(\varphi) \sum_{n=-\infty}^{+\infty} j_{n}^{\prime}\left(k_{\perp} R\right) F_{\mathbf{k}}^{n}(\varphi, t), \\
& F_{\mathbf{k}}^{n}(\varphi, t)=\int_{\tau_{\mathrm{i}}}^{\tau_{\mathrm{i}}+\omega t} d \tau \exp \left[i\left(c k_{0} \omega^{-1}-n\right) \tau\right],
\end{aligned}
$$

we can rewrite (49) as follows:

$$
\begin{aligned}
& y_{\mathbf{k} 1}=\frac{i q \cot \theta}{\sqrt{k_{0}^{3}(2 \pi)^{2} \hbar c}} Y_{\mathbf{k}}(\varphi) \sum_{n=-\infty}^{+\infty} n j_{n}\left(k_{\perp} R\right) F_{\mathbf{k}}^{n}(\varphi, t), \\
& y_{\mathbf{k} 2}=-\frac{q R}{\sqrt{k_{0}(2 \pi)^{2} \hbar c}} Y_{\mathbf{k}}(\varphi) \sum_{n=-\infty}^{+\infty} j_{n}^{\prime}\left(k_{\perp} R\right) F_{\mathbf{k}}^{n}(\varphi, t) .
\end{aligned}
$$

Now, we can calculate the corresponding probabilities $\left|y_{\mathbf{k} \lambda}\right|^{2}$,

$$
\begin{aligned}
& \left|y_{\mathbf{k} 1}\right|^{2}=\frac{q^{2}}{\hbar c} \frac{\cot ^{2} \theta}{k_{0}^{3}(2 \pi)^{2}}\left|\sum_{n=-\infty}^{+\infty} n j_{n}\left(k_{\perp} R\right) F_{\mathbf{k}}^{n}(\varphi, t)\right|^{2}, \\
& \left|y_{\mathbf{k} 2}\right|^{2}=\frac{q^{2}}{\hbar c} \frac{R^{2}}{k_{0}(2 \pi)^{2}}\left|\sum_{n=-\infty}^{+\infty} j_{n}^{\prime}\left(k_{\perp} R\right) F_{\mathbf{k}}^{n}(\varphi, t)\right|^{2} .
\end{aligned}
$$

The radiated energy (39) has to be calculated in the following manner:

$$
\begin{aligned}
& W(1, t)=W_{1}(1, t)+W_{2}(1, t) \\
& W_{1}(1, t)=\hbar c \int d \mathbf{k} k_{0}\left|y_{\mathbf{k} 1}\right|^{2}=\int d \mathbf{k} \frac{q^{2} \cot ^{2} \theta}{k_{0}^{2}(2 \pi)^{2}}\left|\sum_{n=-\infty}^{+\infty} n j_{n}\left(k_{\perp} R\right) F_{\mathbf{k}}^{n}(\varphi, t)\right|^{2}, \\
& W_{2}(1, t)=\hbar c \int d \mathbf{k} k_{0}\left|y_{\mathbf{k} 2}\right|^{2}=\int d \mathbf{k} \frac{q^{2} R^{2}}{(2 \pi)^{2}}\left|\sum_{n=-\infty}^{+\infty} j_{n}^{\prime}\left(k_{\perp} R\right) F_{\mathbf{k}}^{n}(\varphi, t)\right|^{2} .
\end{aligned}
$$

Note that the functions $F_{\mathbf{k}}^{n}(\varphi, t)$ can be represented as:

$$
F_{\mathbf{k}}^{n}(\varphi, t)=\omega \exp \left[-i\left(c k_{0} \omega^{-1}-n\right) \varphi\right] \exp \left[i \frac{\pi}{2}\left(c k_{0} \omega^{-1}-n\right)\right] \int_{0}^{t} d t^{\prime} \exp \left[i\left(c k_{0}-n \omega\right) t^{\prime}\right]
$$


Using the well-known integral representation of Kronecker's delta function

$$
\oint d \varphi \exp \left[i\left(n-n^{\prime}\right) \varphi\right]=2 \pi \delta_{n n \prime}
$$

we can transform the quantities $W_{1}(1, t)$ and $W_{2}(1, t)$ as follows:

$$
\begin{aligned}
& W_{1}(1, t)=q^{2} \omega^{2} \sum_{n=-\infty}^{+\infty} \int_{0}^{\infty} \frac{d k_{0}}{2 \pi} \int_{0}^{\pi} \sin \theta d \theta \cot ^{2} \theta n^{2} j_{n}^{2}\left(k_{\perp} R\right)\left|\int_{0}^{t} d t^{\prime} \exp \left[i\left(c k_{0}-n \omega\right) t^{\prime}\right]\right|^{2} \\
& W_{2}(1, t)=q^{2} \omega^{2} R^{2} \sum_{n=-\infty}^{+\infty} \int_{0}^{\infty} \frac{d k_{0}}{2 \pi} \int_{0}^{\pi} \sin \theta d \theta k_{0}^{2} j_{n}^{\prime 2}\left(k_{\perp} R\right)\left|\int_{0}^{t} d t^{\prime} \exp \left[i\left(c k_{0}-n \omega\right) t^{\prime}\right]\right|^{2}
\end{aligned}
$$

Then the the energy $W(1, t)$ reads:

$$
W(1, t)=\frac{q^{2} \omega^{2}}{2 \pi} \sum_{n=-\infty}^{+\infty} \int_{0}^{\infty} d k_{0} \int_{0}^{\pi} \sin \theta d \theta\left[n^{2} j_{n}^{2}\left(k_{\perp} R\right) \cot ^{2} \theta+k_{0}^{2} R^{2} j_{n}^{\prime 2}\left(k_{\perp} R\right)\right]\left|\int_{0}^{t} d t^{\prime} \exp \left[i\left(c k_{0}-n \omega\right) t^{\prime}\right]\right|^{2} .
$$

\subsection{Derivation of the Schott formula}

Let us study the time behavior of the energy $W(1, t)$ of the one-photon emission (56). One can see that at $t \rightarrow \infty$ this quantity as a function of time is not well defined. However, a real physical meaning has the rate $w(t)$ of the energy emission, which is the time derivative of $W(1, t)$,

$$
\begin{aligned}
& w(t)=\partial_{t} W(1, t)=\frac{q^{2} \omega^{2}}{2 \pi} \sum_{n=-\infty}^{+\infty} K(t) \int_{0}^{\infty} d k_{0} \int_{0}^{\pi} \sin \theta\left[n^{2} j_{n}^{2}\left(k_{\perp} R\right) \cot ^{2} \theta+k_{0}^{2} R^{2} j_{n}^{\prime 2}\left(k_{\perp} R\right)\right] d \theta, \\
& K(t)=\frac{\partial}{\partial t}\left|\int_{0}^{t} d t^{\prime} \exp \left[i\left(c k_{0}-n \omega\right) t^{\prime}\right]\right|^{2} .
\end{aligned}
$$

To compare with the Schott result, we have to consider $w(t)$ as $t \rightarrow \infty$. In fact the problem is reduced to calculating the $\lim _{t \rightarrow \infty} K(t)$. This limit can be easily calculated,

$$
\lim _{t \rightarrow \infty} K(t)=\lim _{t \rightarrow \infty} \frac{2 \sin \left(c k_{0}-n \omega\right) t}{c k_{0}-n \omega}=2 \pi \delta\left(c k_{0}-n \omega\right),
$$

see, e.g., [7]. Taking Eq. (58) into account and the fact that the delta-function in the RHS of Eq. (58) vanishes for negative $n$, we obtain:

$$
\lim _{t \rightarrow \infty} w(t)=\frac{q^{2} \omega^{2}}{c} \sum_{n=1}^{+\infty} n^{2} \int_{0}^{\pi} \sin \theta\left[j_{n}^{2}\left(\frac{n \omega R}{c} \sin \theta\right) \cot ^{2} \theta+\frac{\omega^{2} R^{2}}{c^{2}} j_{n}^{\prime 2}\left(\frac{n \omega R}{c} \sin \theta\right)\right] d \theta .
$$

The result (59) reproduces literally the Schott formula for the rate of the energy radiation by a classical current.

\subsection{Schwinger calculations of the one-photon radiation}

Schwinger in his work [5] considered classical SR, using the method is based on an examination of the energy transfer rate from the electron to the electromagnetic field. Later in Ref. 6] he calculated the quantum corrections of the first order in $\hbar$ to the classical formula, taking into account the quantum nature of the radiating particle but neglecting its spin properties. In 1973 he reexamined the problem, utilizing the source theory to obtain the quantum expression for the spectral distribution of the radiated power [9].

In [5], he presented several different distributions of the instantaneous power. Among them is expression for the power radiated into a unit solid angle about the direction $\mathbf{n}=(\cos \varphi \cos \theta, \sin \varphi \cos \theta, \sin \theta)$ and $\operatorname{contained}$ in a unit angular frequency interval about the frequency $c k_{0}$,

$$
\begin{aligned}
& P\left(\mathbf{n}, k_{0}\right)=\sum_{n=1}^{\infty} \delta\left(c k_{0}-n \omega\right) P_{n}(\mathbf{n}) \\
& P_{n}(\mathbf{n})=\frac{\omega^{2} R}{c^{2}} \frac{q^{2}}{2 \pi} n^{2}\left[\frac{\omega^{2} R^{2}}{c^{2}} j_{n}^{\prime 2}\left(\frac{n \omega R}{c} \cos \theta\right)+\frac{\sin ^{2} \theta}{\cos ^{2} \theta} j_{n}\left(\frac{n \omega R}{c} \cos \theta\right)\right] .
\end{aligned}
$$

The total radiated power can be calculated as

$$
P=\int_{0}^{\infty} c d k_{0} \int P\left(\mathbf{n}, k_{0}\right) d \Omega .
$$


Considering the high-frequency radiation,

$$
1-\frac{\omega^{2} R^{2}}{c^{2}} \ll 1, \theta \ll 1, n \gg 1
$$

and using the connection between the Airy and Bessel functions, Schwinger obtained an alternative representation for his result in the form

$$
\begin{aligned}
& P_{n}(\mathbf{n})=\frac{q^{2} \omega}{6 \pi^{2} R} n^{2}\left(1-\omega^{2} R^{2} / c^{2}+\theta^{2}\right)^{2}\left[K_{2 / 3}^{2}(\zeta)+\frac{\theta^{2} K_{1 / 3}^{2}(\zeta)}{1-\omega^{2} R^{2} / c^{2}+\theta^{2}}\right] \\
& \zeta=\frac{n}{n_{c}}\left(\frac{1-\omega^{2} R^{2} / c^{2}+\theta^{2}}{1-\omega^{2} R^{2} / c^{2}}\right)^{3 / 2},
\end{aligned}
$$

and $n_{c}$ is a critical harmonic number [5]. Note that formal difference in angular distribution between (60) and (59) appear due to different notation and does not lead to any differences in final values.

In Ref. 6] he considered the quantum corrections of the first order in $\hbar$ to the classical formula, taking into account the quantum nature of the radiating electron. In his consideration he neglected the spin properties as at this level of accuracy, the spin degrees of freedom play no role for unpolarized particles. The first-order in $\hbar$ correction to the classical formula (61) can be obtained from the classical expression for the differential radiation probability $\left(c k_{0}\right)^{-1} P\left(\mathbf{n}, c k_{0}\right)$ [6] by making the substitution

$$
c k_{0} \rightarrow c k_{0}\left(1+\frac{\hbar c k_{0}}{E}\right) \text {. }
$$

The total radiated power with the first order quantum corrections obtained by Schwinger reads

$$
w=\frac{2}{3} \omega \frac{q^{2}}{R}\left(\frac{E}{m c^{2}}\right)^{4}\left[1-\sqrt{3} \frac{55}{16} \frac{\hbar}{m c R}\left(\frac{E}{m c^{2}}\right)^{2}+O\left(\hbar^{2}\right)\right]
$$

In Ref. 9] Schwinger considered the radiation of a spinless charged particle in the homogeneous magnetic field, and obtained the spectral distribution of the radiated power $w\left(k_{0}\right)$ (here $c=\hbar=1$ ) in the form

$$
w\left(k_{0}\right)=\frac{c k_{0} q^{2}}{\pi m} \frac{m^{2}}{E^{2}}\left\{\int_{0}^{\infty} \frac{d x}{x}\left(1+2 x^{2}\right) \sin \left[\frac{c k_{0}}{\omega}\left(\frac{m}{E}\right)^{3}\left(x-\frac{x^{3}}{3}\right)\right]-\frac{1}{2} \pi\right\}, x=\frac{1}{2} \omega t \frac{E}{m} .
$$

According to the author, Eq. (66) in the classical limit reproduces the Schott formula.

Note that the formulas (67) and (66) include both the corrections due to electron recoil and the effects of quantization of the electromagnetic field. As for the comparison with our result, the angular distributions coincide with the Schott formula and are not affected by quantum corrections.

\subsection{One-photon radiation of scalar particles due to transitions between Landau levels}

When presenting the results obtained by other authors, we use the same system of units that was utilized in the cited articles.

There is a different approach to calculation of radiation of the spinless charged particle in due to one-photon transitions between the energy levels presented in Ref. 24. These calculations are based on the exact solutions of the Klein-Gordon equation in the uniform magnetic field (the Furry picture approach). The spectral angular distribution of the radiated power in this approach has the form

$$
\begin{aligned}
& w=\frac{27}{16 \pi^{2}} w_{0} \xi^{2} \varepsilon_{0}^{-5 / 2} \int_{0}^{\infty} d y \int_{0}^{\pi} \frac{\sin \theta d \theta}{(1+\xi y)^{3}} y^{2}\left[\varepsilon^{2} K_{2 / 3}^{2}\left(z_{0}\right)+\varepsilon \cos \theta K_{1 / 3}^{2}\left(z_{0}\right)\right] \\
& w_{0}=\frac{8}{27} \frac{q^{2} m^{2} c^{2}}{\hbar^{2}}, \xi=\frac{3}{2} \frac{e \hbar H}{m^{2} c^{3}} \frac{E}{m c^{2}}, \varepsilon_{0}=\left(\frac{m c^{2}}{E}\right)^{2} \\
& z_{0}=\frac{y}{2}\left(\frac{\varepsilon}{\varepsilon_{0}}\right)^{3 / 2}, \varepsilon=1-\frac{\omega^{2} R^{2}}{c^{2}} \sin ^{2} \theta, E=\frac{m c^{2}}{\sqrt{1-\omega^{2} R^{2} / c^{2}}}
\end{aligned}
$$

where $K_{n}\left(z_{0}\right)$ are Airy functions, and $E$ is the electron energy. Unfortunately, no representation of the (67) in terms of the Bessel functions is given by the authors; however, it is claimed that Eq. (67) in the limit $\hbar \rightarrow 0$ reproduces the classical result. 


\section{Two-photon radiation}

The probability $p(2, t)$ and the energy $W(2, t)$ of the two-photon radiation for a circular current (40) have the form:

$$
\begin{aligned}
& p(2, t)=\frac{\alpha^{2}}{(2 \pi)^{2}}\left\{\int \frac{d \mathbf{k}}{2 k_{0}}\left[k_{0}^{-2} F_{1}(\mathbf{k}, t) \cot ^{2} \theta+R^{2} F_{2}(\mathbf{k}, t)\right]\right\}^{2}, \\
& W(2, t)=\frac{\alpha^{2} \hbar c}{(2 \pi)^{2}}\left\{\int d \mathbf{k}\left[k_{0}^{-2} F_{1}(\mathbf{k}, t) \cot ^{2} \theta+R^{2} F_{2}(\mathbf{k}, t)\right]\right\} \\
& \times\left\{\int \frac{d \mathbf{k}^{\prime}}{k_{0}^{\prime}}\left[k_{0}^{\prime-2} F_{1}\left(\mathbf{k}^{\prime}, t\right) \cot ^{2} \theta^{\prime}+R^{2} F_{2}\left(\mathbf{k}^{\prime}, t\right)\right]\right\},
\end{aligned}
$$

where

$$
F_{1}(\mathbf{k}, t)=\left|\sum_{n=-\infty}^{+\infty} n j_{n}\left(k_{\perp} R\right) F_{\mathbf{k}}^{n}(\varphi, t)\right|^{2}, F_{2}(\mathbf{k}, t)=\left|\sum_{n=-\infty}^{+\infty} j_{n}^{\prime}\left(k_{\perp} R\right) F_{\mathbf{k}}^{n}(\varphi, t)\right|^{2} .
$$

It is useful to compare our results with the calculations of two-photon radiation presented in other works. In the Ref. 25, it was considered the bremsstrahlung of relativistic electrons in the so-called approximation of soft photons (the total energy of emitted photons is much less than the energy of a relativistic electron). Our initial assumption, that the classical current $J(x)$ remains unchanged, despite the radiation losses matches with this approximation. The authors of Ref. 25] had used the expression for the instantaneous spectral distribution of the radiation energy of an electron using the Liénard-Wiechert potentials. In such a way they have obtained the total electromagnetic energy of the one-photon radiation. If the electric current in the latter quantity is taken in the form (40), it coincides with our result $W(1, t)$ given by Eq. (56). Then the probability of emitting a photon is defined by the authors as $p(\{1\}, t)=W(\{1\}, t) /\left(\hbar c k_{0}\right)$ [here $W(\{1\}, t)$ is the integrand of $W(1, t)$ ] and the probability $p(\{N\}, t)$ of emitting $\{N\}$ soft photons in a narrow range of angles along the electron motion direction reads:

$$
p(\{N\}, t)=\prod_{i=1}^{N} p\left(1_{\mathbf{k}_{i} \lambda_{i}}, t\right)=\prod_{i=1}^{N}\left|y_{\mathbf{k}_{i} \lambda_{i}}\right|^{2} .
$$

According to the authors, "when integrating in a finite interval of frequencies and directions, one must to introduce a factor $(N !)^{-1}$ that takes into account the identity of the photons". Thus, they arrive to our result (31), which contains such factor for any momenta $\mathbf{k}$ without heuristic prescriptions. It is easy to verify that using the same approximation of the small difference between the angles $\varphi_{1}$ and $\varphi_{2}$ of photons emitted, $\Delta \varphi=\left(\varphi_{1}-\varphi_{2}\right) \ll 1$, we obtain from Eq. (31) for the probability of the two-photon radiation the following result:

$$
p(2, t)=\frac{25}{24} \alpha^{2} \omega \gamma \Delta \varphi, \gamma=\left(1-\omega^{2} R^{2} / c^{2}\right)^{-1 / 2}
$$

It coincides with the one of the work [25].

It should be noted that in Refs. [12 and [11, the authors calculated two-photon synchrotron emission, considering electron transitions between Landau levels by the help of the corresponding solutions of the Dirac equation. In the approximation accepted in the work [25] they derived corrections to Eq. (71) of the order $\hbar$ due to the quantum nature of the electron and due to its spin.

\section{Concluding remarks}

As was said in the Introduction, in the beginning, the SR was studied by classical methods using the LiénardWiechert potentials of electric currents. Subsequently, it became clear that in some cases, quantum corrections to classical results may be important. These corrections were studied, considering the emission of photons arising from electronic transitions between spectral levels, described in terms of the Dirac equation. In this paper, we have considered an intermediate approach, in which electric currents generating the radiation are treated classically, whereas the quantum nature of the radiation is taken into account exactly. Such an approximate approach allows one to study the one-photon and multi-photon radiation without complicating calculations using corresponding solutions of the Dirac equation. We have constructed exact quantum states (8) of the electromagnetic field interacting with classical currents and studied their properties. By their help, we have calculated a probability of photon emission by classical currents from the vacuum initial state and obtained relatively simple general formulas for the one-photon and multi-photon radiation. Using the specific circular electric current, we have calculate the corresponding one-photon and two-photon SR. It was demonstrated that the emitted single-photon power per unit time in the limit $t \rightarrow \infty$ coincides with the classical expression obtained by Schott. This is not strange, since Schott's result was already semi-classical, since he treated the electromagnetic field in terms of the Maxwell's equations. It is well known that, see e.g. [16], in fact, the Maxwell equations can be interpreted as the Schrödinger equation for a single photon, the absence of the Planck constant $\hbar$ in these equations as well as in the Schott formula is associated with the masslessness of the photon. The consideration of the electromagnetic radiation in a semiclassical manner, using Maxwell's 
equations, often allows one to study quantum effects the of radiation [26]. Schwinger's calculations of SR contain $\hbar$ since he used elements of QFT that take into account quantum character of electron motion and in the limit $\hbar \rightarrow 0$ lead to the Schott result. The same situation takes place with calculations of the SR radiation of a spinless charged particle due to transitions between energy levels with one-photon emission presented in Ref. [24]. The proposed approach provides an opportunity to separate the effects of radiation associated with the quantum nature of the electromagnetic field from the effects caused by the quantum nature of the electron. The calculation of multiphoton corrections is significantly simplified compared, for example, with the approach described in [11, 12, 25], where a two-photon correction to the radiation of an electron moving in a circular orbit in a constant uniform magnetic field is calculated within the framework of the Furry picture. Finally, it becomes possible to study the initial states of the system other than the vacuum initial state (the state without initial photons). Using these state vectors, the probabilities $p(N, t)(32)$ and the energy $W(N, t)$ (36) of $N$ photon radiation induced by classical currents are derived. The latter quantity can be summed exactly representing the total energy $W(t)$ (38) of emitted photons. The obtained results can be used for the systematic study of the multiphoton SR.

\section{Acknowledgements}

Bagrov acknowledges support from Tomsk State University Competitiveness Improvement Program. Gitman is supported by the Grant No. 2016/03319-6, Fundação de Amparo à Pesquisa do Estado de São Paulo (FAPESP), and permanently by Conselho Nacional de Desenvolvimento Científico e Tecnológico (CNPq). The work of Shishmarev was supported by the Russian Foundation for Basic Research (RFBR), project number 19-32-60010.

\section{Appendix}

Here we show that the sum (37) can be calculated analytically with the help of representation (35). We start at the definition of $W(N, t)$ from Eq. (35),

$$
W(N, t)=\hbar c(N !)^{-1} \sum_{\lambda_{1}=1}^{2} \sum_{\lambda_{2}=1}^{2} \ldots \sum_{\lambda_{N}=1}^{2} \int d \mathbf{k}_{1} d \mathbf{k}_{2} \ldots d \mathbf{k}_{N}\left[\sum_{j=1}^{N}\left|\mathbf{k}_{j}\right|\right] \prod_{i=1}^{N}\left|y_{\mathbf{k}_{i} \lambda_{i}}\right|^{2} .
$$

We first consider the term with $j=1$. In the entire integrand (A1), only the factor $\left|\mathbf{k}_{1}\right|\left|y_{\mathbf{k}_{1} \lambda_{1}}\right|^{2}$ depends on $\lambda_{1}$ and $\mathbf{k}_{1}$. Therefore, everything except the factor $\left|\mathbf{k}_{1}\right|\left|y_{\mathbf{k}_{1} \lambda_{1}}\right|^{2}$ can be taken out from the signs of the sum over $\lambda_{1}$ and the integral over $d \mathbf{k}_{1}$. Since the indices $i$ are dumb (the limits of all summations and integrations are the same), we can cyclically shift their numbering $(i \rightarrow i-1$, i.e., $2 \rightarrow 1,3 \rightarrow 2, \ldots, N \rightarrow N-1,1 \rightarrow N)$. We do the same with each term from the $\operatorname{sum} j=2,3,4, \ldots, N-1$. Now it's obvious that the sum over $j$ in (A1) degenerates into a factor $N$, and the quantity $W(N, t)$ takes the form:

$$
W(N, t)=\frac{\hbar c}{(N-1) !} \sum_{\lambda_{1}=1}^{2} \sum_{\lambda_{2}=1}^{2} \ldots \sum_{\lambda_{N}=1}^{2} \int d \mathbf{k}_{1} d \mathbf{k}_{2} \ldots d \mathbf{k}_{N}\left|\mathbf{k}_{N}\right| \prod_{i=1}^{N}\left|y_{\mathbf{k}_{i} \lambda_{i}}\right|^{2}
$$

It is easy to see that Eq. (A2) can be written as:

$$
W(N, t)=\frac{\hbar c}{(N-1) !} \sum_{\lambda_{N}=1}^{2} \int d \mathbf{k}_{N}\left|\mathbf{k}_{N}\right|\left|y_{\mathbf{k}_{N} \lambda_{N}}\right|^{2} \prod_{i=2}^{N}\left[\sum_{\lambda_{i}=1}^{2} \int d \mathbf{k}_{i}\left|y_{\mathbf{k}_{i} \lambda_{i}}\right|^{2}\right] .
$$

Finally, getting rid of dumb indices, we obtain:

$$
\begin{aligned}
& W(N, t)=\frac{\hbar c A}{(N-1) !}\left[\sum_{\lambda=1}^{2} \int d \mathbf{k}\left|y_{\mathbf{k} \lambda}\right|^{2}\right]^{N-1}, \\
& A=\sum_{\lambda=1}^{2} \int d \mathbf{k} k_{0}\left|y_{\mathbf{k} \lambda}\right|^{2}, k_{0}=|\mathbf{k}| .
\end{aligned}
$$

The total energy $W(t)$ reads:

$$
W(t)=\sum_{N=1}^{\infty} W(N, t)=\hbar c A \sum_{N=1}^{\infty}[(N-1) !]^{-1}\left[\sum_{\lambda=1}^{2} \int d \mathbf{k}\left|y_{\mathbf{k} \lambda}\right|^{2}\right]^{N-1}
$$

The sum over $N$ can be reduced to an exponent by the change $N=M-1$. Thus, we justify Eq. (38). 


\section{References}

[1] F. R. Elder, A. M. Gurevitch, R. V. Langmuir and A. C. Pollock, Phys. Rev. 71, 829 (1947).

[2] L. D. Landau and E. M. Lifshitz, The classical theory of fields, (Pergamon Press, Oxford, 1971).

[3] J. D. Jackson, Classical Electrodynamics, 3rd Edition (J. Wiley \& Sons, New York, 1998).

[4] G. A. Schott, Phil. Mag. 13, 657 (1907); Ann. Phys. 329, 635 (1907); Electromagnetic Radiation (Cambrige University Press, Cambrige, 1912).

[5] J. Schwinger, Phys. Rev. 75 (12), 1912 (1949).

[6] J. Schwinger, Proc. Nat. Acad. Sci. U. S. 40, 132 (1954).

[7] A. A. Sokolov, I. M. Ternov, Sov. Phys. JETP 4, 396 (1957); Synchrotron Radiation (Academic Verlag, Berlin, 1968); Radiation from relativistic electrons (American Institute of Physics, New York, 1986).

[8] J. Schwinger, Particles, Sources, and Fields, Vol. 1 (1970) Vol. 2 (1973) (Addison-Wesley).

[9] J. Schwinger, Phys. Rev. D 7 (6), 1696 (1973).

[10] A. A. Sokolov, I. M. Ternov, Proc. Int. Conf. on High Energy Accelerators, 21 (1963); Dokl. Akad. Nauk USSR 153, 1053 (1963).

[11] A. A. Sokolov, A. M. Voloshenko, V. Ch. Zhukovskii and Yu. G. Pavlenko, Sov. Phys. Journ. 9, 46 (1976).

[12] A. A. Sokolov, A. M. Voloshenko, V. Ch. Zhukovskii and Yu. G. Pavlenko, Russ. Phys. Journ. 19, 1139 (1976).

[13] W. Heitler, The Quantum Theory of Radiation, (Oxford Univ. Press, London, 1936).

[14] S. Schweber, An Introduction to Relativistic Quantum Field Theory (Harper \& Row, New York, 1961).

[15] N. N. Bogoliubov and D. V. Shirkov, Introduction to the Theory of Quantized Fields, 3-rd ed. (John Wiley \& Sons, New York, 1980).

[16] A. I. Akhiyeser and V. B. Berestetskii, Quantum Electrodynamics (Science, Moscow, 1981).

[17] D. M. Gitman and I. V. Tyutin, Canonical quantization of fields with constraints (Nauka, Moscow, 1986); Quantization of Fields with Constraints (Springer-Verlag, Berlin, 1990).

[18] V. G. Bagrov, D. M. Gitman, V. A. Kuchin, External field in quantum electrodynamics and coherent states, in Actual problems of Theoretical physics, M. V. Lomonosov Moscow State University, Moscow, Russia, pp. 334-342 (1976); Sov. Phys. Journ. 4, 152 (1974).

[19] V. G. Bagrov, D. M. Gitman, A. D. Levin, J. Russ. Laser. Res. 32, 317 (2011).

[20] N. N. Bogoliubov, D. V. Shirkov, Quantum Fields (Nauka, Moscow 1980).

[21] D. V. Galtsov, Yu. V. Gratz and V. Ch. Zhukovsky, Classical fields (Moscow State University Press, Moscow, 1991).

[22] R. P. Feynman, Phys. Rev. 84, 108 (1951).

[23] R. J. Glauber, Phys. Rev. 84, 1 (1951).

[24] Radiation Theory of Relativistic Particles, Editor: V. A. Bordovitsyn, (Fizmatlit, Moscow, 2002); V. G. Bagrov, Izv. VUZov. Fizika 5, 121 (1965).

[25] A. M. Voloshchenko, V. Ch. Zhukovskii, and Yu. G. Pavlenko, Moscow University Physics Bulletin 31, 42 (1976).

[26] E. T. Jaynes, F. W. Cummings, Proc. IEEE 51, 89 (1963). 